\title{
Gallbladder cancer: review of a rare orphan gastrointestinal cancer with a focus on populations of New Mexico
}

\author{
Jacklyn M. Nemunaitis ${ }^{1,2+}$, Ursa Brown-Glabeman ${ }^{1,2+}$, Heloisa Soares ${ }^{1,2}$, Jessica Belmonte ${ }^{1,2}$, Ben Liem,2, \\ Itzhak Nir ${ }^{1,3}$, Victor Phuoc ${ }^{1,3}$ and Rama R. Gullapalli ${ }^{1,4,5^{*}+}$ (D)
}

\begin{abstract}
Gallbladder cancer is a rare malignancy of the biliary tract with a poor prognosis, frequently presenting at an advanced stage. While rare in the United States overall, gallbladder cancer has an elevated incidence in geographically distinct locations of the globe including Chile, North India, Korea, Japan and the state of New Mexico in the United States. People with Native American ancestry have a much elevated incidence of gallbladder cancer compared to Hispanic and non-Hispanic white populations of New Mexico. Gallbladder cancer is also one of the few bi-gendered cancers with an elevated female incidence compared to men. Similar to other gastrointestinal cancers, gallbladder cancer etiology is likely multi-factorial involving a combination of genomic, immunological, and environmental factors. Understanding the interplay of these unique epidemiological factors is crucial in improving the prevention, early detection, and treatment of this lethal disease. Previous studies have failed to identify a distinct genomic mutational profile in gallbladder cancers, however, work to identify promising clinically actionable targets is this form of cancer is ongoing. Examples include, interest in the HER2/Neu signaling pathway and the recognition that chronic inflammation plays a crucial role in gallbladder cancer pathogenesis. In this review, we provide a comprehensive overview of gallbladder cancer epidemiology, risk factors, pathogenesis, and treatment with a specific focus on the rural and Native American populations of New Mexico. We conclude this review by discussing future research directions with the goal of improving clinical outcomes for patients of this lethal malignancy.
\end{abstract}

Keywords: Gallbladder Cancer, New Mexico, Gallstones, Personalized medicine, HER2/Neu, Chronic Inflammation, Heavy Metals

\section{Background}

Gallbladder cancer $(\mathrm{GBC})$ is a rare malignant neoplasm [1]. It is, however, the most common malignancy of the biliary tract and sixth in overall gastrointestinal cancer incidence [1, 2]. GBC is an aggressive, often lethal malignancy with a mean overall survival of six months $[1,2]$. The disease is rapidly progressive and rarely diagnosed at an early stage, contributing to poor clinical outcomes.

\footnotetext{
* Correspondence: RGullapalli@salud.unm.edu

${ }^{\dagger}$ Jacklyn M. Nemunaitis, Ursa Brown-Glabeman and Rama R. Gullapalli contributed equally to this work.

${ }^{1}$ Comprehensive Cancer Center, University of New Mexico, Albuquerque, NM, USA ${ }^{4}$ Department of Pathology, University of New Mexico Health Sciences Center, Albuquerque, NM, USA

Full list of author information is available at the end of the article
}

The worldwide incidence of GBC is less than $2 / 100,000$ individuals and there is considerable variation in this number based on geographic distribution and gender [3]. This variability is likely due to a combination of environmental and genetic factors. Women have a higher incidence ratio (F:M ratio $\sim 2.6: 1$ ), with typical onset in the sixth decade. Inordinately high rates of GBC occur among Native Americans and Southeast Asians, with several other scattered geographical locations of elevated GBC incidence across the world $[1,4,5]$. While the disease is rare overall in the United States, Native Americans in New Mexico have uniquely high rates of GBC [4]. Native American women are more commonly affected compared to Native American men, in line with globally observed trends [4].

(c) The Author(s). 2018 Open Access This article is distributed under the terms of the Creative Commons Attribution 4.0 International License (http://creativecommons.org/licenses/by/4.0/), which permits unrestricted use, distribution, and reproduction in any medium, provided you give appropriate credit to the original author(s) and the source, provide a link to the Creative Commons license, and indicate if changes were made. The Creative Commons Public Domain Dedication waiver (http://creativecommons.org/publicdomain/zero/1.0/) applies to the data made available in this article, unless otherwise stated. 
Due to the rarity of this cancer, GBC is an orphan disease with minimal research efforts currently (both basic and translational) in the United States. Currently, no targeted therapies exist for the treatment of $\mathrm{GBC}$ in routine clinical practice [6]. A better understanding of GBC pathogenesis is urgently needed to develop targeted therapy approaches to improve outcomes in GBC patients. In this review, we discuss the pathogenesis of $\mathrm{GBC}$, the current status of $\mathrm{GBC}$ therapies, the future directions with a focus on GBC pathogenesis in rural and Native American populations of New Mexico.

\section{Epidemiology and risk factors for gallbladder cancer}

The geographic distribution of GBC incidence is highly uneven, with remarkable rarity in certain parts of the world, and near epidemic rates in others [5]. This uneven incidence distribution is likely due to a combination of genetic and local environmental factors. The highest rates of GBC are found in Latin America and Asia and the lowest rates are seen in the United States and parts of Western and Mediterranean European countries [7] . For example, among Chilean women, GBC is the leading cause of cancer death, with mortality rates higher than those for breast or lung [3, 5]. Other high-risk areas around the world include Poland (14/100,000), northern India (21.5/100,000 for women), south Pakistan (11.3/ $100,000)$, Israel $(5 / 100,000)$, and Japan $(7 / 100,000)$ [1].

Interestingly, the highest rates of $\mathrm{GBC}$ are generally found in indigenous Amerindian populations of the Americas (e.g., Chile, Bolivia, and states such as New Mexico in the United States). Among Native Americans in the United States (including Alaska Natives) the rate of GBC incidence is $3.3 / 100,000$ [8] compared to the $0.4-1.5 / 100,000$ incidence rate seen in the non-Native American populations. Native Americans from the state of New Mexico have the highest rates of GBC incidence in the United States [4]. The elevated GBC incidence is observed in Native American women and men compared to the other ethnicities in New Mexico (Caucasians and Hispanics in New Mexico) [4]. Interestingly, the Hispanic populations of New Mexico also show an elevated incidence of GBC (compared to Caucasians in New Mexico), though not to the degree seen in Native Americans [4]. In Chile, for each 1\% increase in Mapuche heritage, an indigenous people common in Chile, there is an associated $3.7 \%$ increase in risk for GBC [9]. These observations argue for specific predisposing genetic factors (direct or indirect) unique to certain indigenous populations.

Numerous risk factors driving GBC have been studied including demographic factors, pre-existing gallbladder disease, and environmental exposures. Predisposing conditions for GBC include chronic gallstones [10], chronic infections leading to cholangitis (e.g., Salmonella enterica typhi and $H$. pylori seen mainly in India and Bangladesh) [11-14], porcelain gallbladder [15], Mirizzi's syndrome [16], bile reflux [17], and gallbladder polyps. Environmental factors such as diet, toxic ingestions, aflatoxins, elevated capsaicin consumption and vitamin deficiencies have all been controversially linked to GBC pathogenesis $[1,6,13,18,19]$. Obesity and poor socio-economic conditions are associated with elevated GBC incidence, though the underlying mechanisms remain uncertain.

The underlying causes of the elevated female incidence of GBC is unknown. Endogenous estrogen plays a key role in gallstone development [20]. Prolonged lifetime estrogen exposure is associated with elevated risk of GBC carcinogenesis [21, 22] through low parity, young age at menarche, late age at first pregnancy, oral contraceptive use, estrogen replacement therapy, and prolonged fertility [20]. A recent meta-analysis of 14 studies found an increased risk of GBC in overweight women (relative risk 1.25), however, a similar relationship was not identified in overweight men [23]. This correlation is interesting given the well-established link between obesity in women and elevated estrogen levels. Definitive biological mechanisms of the numerous risk factors associated with GBC carcinogenesis are lacking currently. This is mainly due to the paucity of readily available cell and animal models of GBC carcinogenesis which remain to be developed.

\section{Gallbladder cancer in New Mexico}

Native American populations of New Mexico have a significantly elevated incidence of GBC compared to Hispanic and non-Hispanic white populations within the state [4]. The state of New Mexico is ethnically diverse with $45 \%$ non-Hispanic whites, $42 \%$ Hispanics and $10 \%$ American Indians. Nir et al. reviewed the SEER cancer registry data from 1981 to 2008 to identify trends of biliary tract cancer incidence (GBC and biliary tract cancers) in New Mexico [4]. Similar to epidemiological patterns seen elsewhere in the world, Nir et al. observed GBC was the commonest malignancy in the biliary tract with a higher incidence among women compared to men across all ethnicities in New Mexico. Native American women showed an eight fold higher GBC incidence compared to non-Hispanic whites while a four fold higher incidence was seen among Hispanic women compared to Caucasian women [4]. Among men, the GBC incidence among the Native Americans, Hispanic and Caucasian ethnicities was 4.1, 1.1 and $0.8 \%$ respectively [4]. We reviewed the publicly available GBC incidence data for this review paper from the New Mexico tumor registry (https://www.cancer-rates.info/nm/) for the years 2000-2014 (see Fig. 1). Consistent with the data from Nir et al. [4], we continue to observe an elevated incidence of 


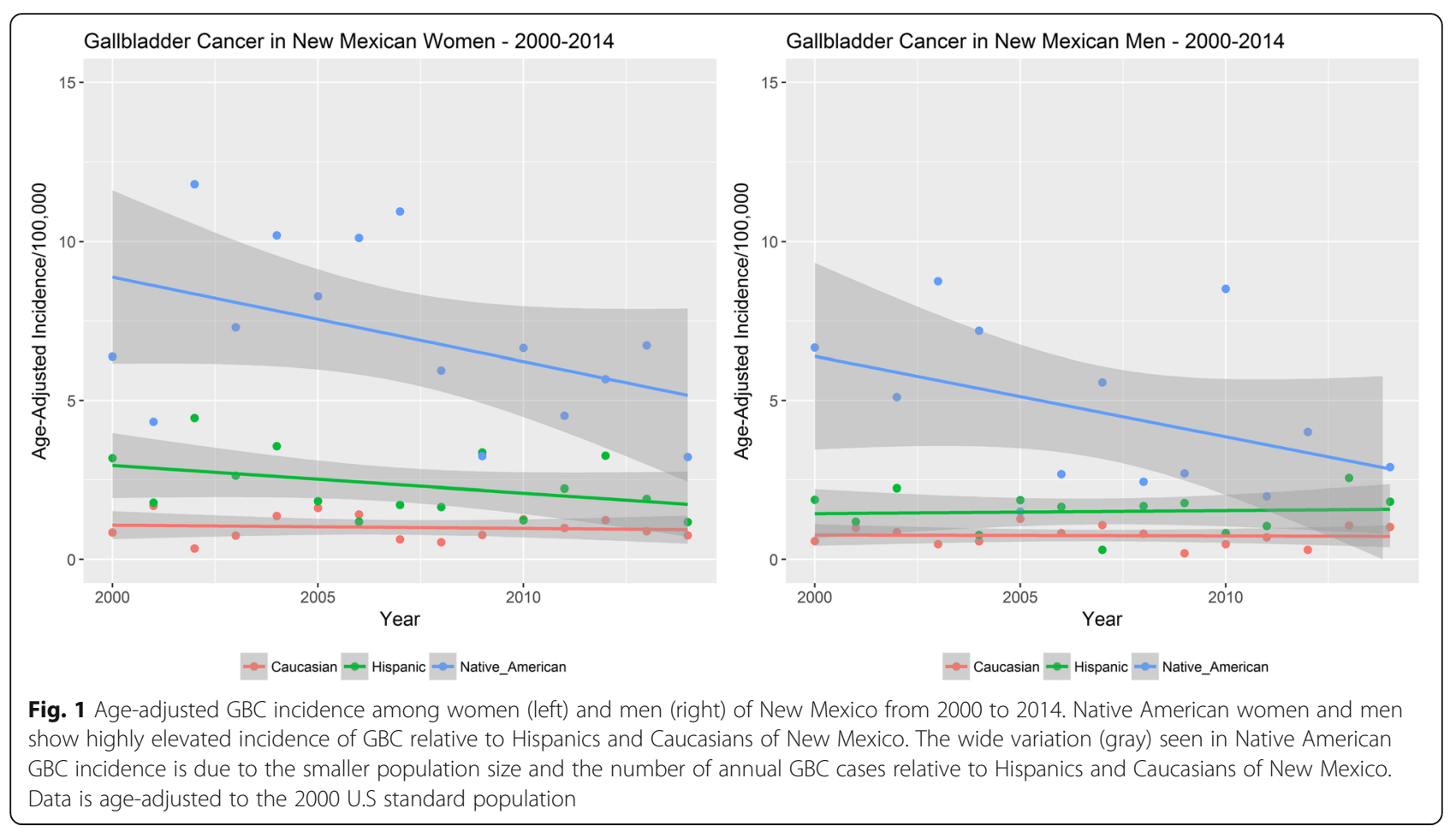

GBC among Native American minorities relative to the Caucasians and Hispanics within the state of New Mexico from 2000 to 2014. The elevated GBC incidence trend is seen in both Native American men and women of the state (Fig. 1). The age adjusted incidence rate of GBC in New Mexico was 6.52, 2.17, and 0.96 per 100,000 among Native American, Hispanic, and Caucasian women respectively. For men, similar age-adjusted GBC incidence rates were 3.73, 1.27 and 0.71 per 100,000 (Native American, Hispanic, and Caucasian).

An elevated prevalence of gallstones amongst Native Americans may be one of the key risk factors associated with the increased GBC development. A prolonged clinical history of gallstones is the single most important risk factor associated with the development of GBC. The Native American populations of the American continent have a unique association with increased gallstone incidence [24]. Gallstone disease was identified as "the native man's burden" by researchers as early as 1960s [25]. Sievers and Marquis first identified the elevated incidence of gallstones among the Native American populations of the South-Western United States, including the Navajo, Zuni and Hopi populations of New Mexico and Arizona [26]. It is now recognized that the incidence of gallstones is high among Native American populations across the entire American continent [24, $25,27]$. Canadian Indians have a $62 \%$ estimated incidence of gallstones. The prevalence of gallstones is approximately $70-80 \%$ among Pima Indian women of the age 25-35 years [25]. Similar patterns of elevated incidence are seen in Native American populations of South America (e.g., Mapuche Indians). In contrast, incidence of gallstones among non-Hispanic white women and men is reported to be 16.6 and $8.6 \%$ respectively [4].

Mortality from GBC in the past few decades has declined in part due to increased surgical intervention for gallstone removal. From 1970 to 1980, the mortality of GBC declined sharply in countries that performed more frequent cholecystectomies. In developed countries including England, Scotland, Canada, United States and Sweden, mortality from GBC is inversely associated with the frequency of cholecystectomy [10]. Alongside the modern plateau in surgical intervention for gallstones, incidence rates of GBC in the entire United States has remained stable [28]. However, in New Mexico the incidence of GBC amongst all ethnic groups, including Native Americans, appears to be gradually declining [4]. Gallstones in the developed world (including the Native Americans) tend to be of the cholesterol type reflecting the role of obesogenic diets [25]. However, Native Americans develop gallstones at an earlier age without a correlation to obesity suggesting a genetic basis of gallstone formation [25]. While the molecular genetics of gallstones have been studied in detail across the world, similar gene association studies in populations of New Mexico are lacking currently.

While gallstones are an important risk factor in GBC carcinogenesis, it does not fully explain the increased risk of GBC. The majority of patients $(\sim 98 \%)$ with gallstones never develop GBC suggesting the need for additional 'hits' in the GBC pathogenesis process. Various environmental 
risk factors have been examined in detail with no clear correlation at the current time [29]. It is very likely the second 'hit' in GBC are risk factors unique to the geographical locations (e.g., Salmonella in India, Aflatoxins in South America [18]). One under-explored hypothesis is the role of heavy metals in GBC pathogenesis. Basu et al. examined the presence of heavy and trace metals in samples of GBC from India [30] and identified significantly elevated copper concentrations in the serum, bile and gallbladder tissue samples of GBC patients [22]. Elevated levels of lead, cadmium, chromium and nickel levels were also seen in the serum and bile of these patients. A second study by Chhabra et al. identified elevated levels of chromium, lead and arsenic in Indian GBC patients compared to GBC patients from Japan [31]. The precise mechanisms by which heavy metals induce carcinogenic transformation in gallbladder remains unknown.

The southwestern portion of United States has a legacy of abandoned mines, the most significant of which is Uranium mining in the Four Corners location of the Navajo Nation [32]. More than 10,000 abandoned mines currently exist in western United States in states of New Mexico, Arizona and Colorado [32]. Many Native Americans live close to these abandoned mines with high levels of heavy metal exposures such as uranium, arsenic and cadmium due to contaminated water supplies in these communities [32-34]. Uranium is a well-known nephrotoxin while arsenic tends to act as co-carcinogen for various other heavy metals [35, 36]. It is possible that heavy metals may act as a "second-hit" acting in synergy with gallstone induced inflammation to drive gallbladder carcinogenesis among Native American populations of New Mexico. Using the publicly available data from the New Mexico Tumor registry, we also mapped the age-adjusted GBC incidence data at a New Mexico county level (see Fig. 2). The geospatial mapping shown in Fig. 2 vividly illustrates the regional preponderance of GBC incidence patterns within the state of New Mexico. The north-west corner of the state is home to the Navajo reservation where a large majority of the New Mexican Native American population currently resides. Unsurprisingly, this region also shows the highest incidence rates of GBC cases in New Mexico (Fig. 2). The Hispanic population is more prevalent in the North East and East portions of the state (adjacent to Texas) with localized increases GBC cases in these areas of the state within that ethnicity. The Caucasian population shows a diffuse, low incidence distribution of GBC across the entire state of New Mexico as expected. There is an increased density of abandoned mines near the Native American reservations (in the north-west part of the state, data not shown) representing an intriguing hypothesis of heavy metal exposure as one of the possible factors driving the elevated GBC causation among Native Americans which needs further study. Studies are currently underway in our lab to examine these exact issues in detail.

\section{Chronic inflammation: The common link of gallbladder cancer pathogenesis}

Extensive evidence supports the role played by chronic inflammation in carcinogenesis in general and in GBC specifically [37]. GBC is a "classic" example of cancer pathogenesis driven by chronic inflammation [38]. Chronic inflammation of the gallbladder may be due to pre-existing gallstones or infections (e.g., Salmonella). A higher level of the acute phase reactant c-reactive protein (CRP) is associated increased risk of GBC [39]. Chronic inflammation induced carcinogenesis involves recurrent cycles of epithelial damage and repair. Long-term chronic

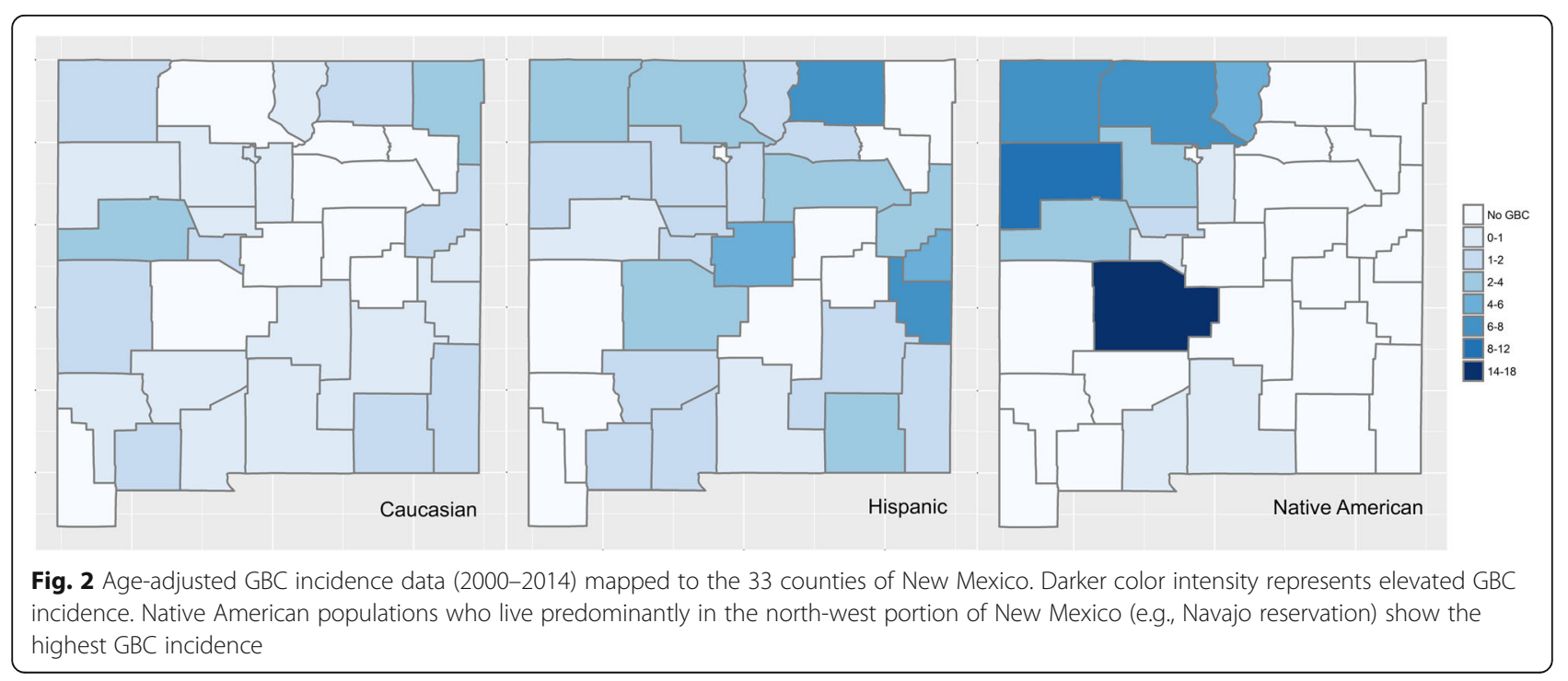


inflammation results in the sustained release of inflammatory mediators, such as cytokines, chemokines, and prostaglandins into the tissue microenvironment. Cytokine induced inflammation may result in activation of oncogenes and inactivation of tumor suppressor genes leading to cell transformation, proliferation, and inhibition of apoptosis [38, 40].

Multiple inflammatory effector molecules have been linked to the development of GBC. Tumor necrosis factor alpha (TNF-a), a major pro-inflammatory cytokine, shows increased expression within the gallbladder mucosa from hyperplasia to carcinoma [41]. The relative expression of TNF-alpha increases with tumor stage [41]. Similarly, the chemokine (CXC motif) ligand 12 and its receptor CXCR4 have been shown to play a role in tumor survival and metastases in GBC [42]. CXCL12 is largely expressed in fibroblasts and is involved in cell proliferation, migration, and invasion. Both CXCL12 and CXCR4 are overexpressed in GBC [42] and has been linked with poor prognosis [43]. Another implicated molecule is cyclooxygenase $2(C O X-2)$. This molecule converts arachidonic acid to prostaglandins, contributing to chronic inflammation. $\mathrm{COX}-2$ has been found to be overexpressed in $91.7 \%$ of GBCs, whereas it is present in only $28.6 \%$ of chronic cholecystitis cases comparatively [44]. In one study, a significant association between the toll-like receptor polymorphisms in the TLR2 and TLR4 genes was associated with GBC [45]. The link between innate immunity and GBC is interesting due to the possible key role played by chronic Salmonella infections and GBC in India. The toll-like receptor genes are key players in identifying lipopolysaccharide elements associated with gram negative bacterial infections. In addition to the innate immunity $T L R$ genes, other significant immune molecule associations with GBC include IL1B, IL10 and CCR5 [46].

Chronic inflammation, via various chemokines, can result in somatic mutations linked with carcinogenesis. Some examples of this process include, TP53, a classic tumor suppressor gene, is the most frequently identified mutation in GBC, with a mutation rate of over $50 \%$ of GBC cases, and clearly plays a role in tumorigenesis [47]. Alterations in TP53 protein expression have been found in histologically normal gallbladders with chronic cholecystitis and the frequency of mutations increases as the pathology progresses to a malignant state [48]. Polymorphisms in $I L-10$ and VEGF genes have been found to be related to higher risk of GBC [49]. MicroRNAs play an important role in both inflammation and cancer. They can act as inflammatory mediators, oncogenes, or tumor suppressors depending on the surrounding environment [50]. Not surprisingly, expression levels of certain miRNA strands in vitro significantly enhanced GBC proliferation and invasion [51]. The strong associations discussed above argue that there may be a role for anti-inflammatory agents in the prevention or treatment of GBC [52, 53]. Population studies have indicated that the use of aspirin, an inhibitor of COX-2 protein, may reduce the risk of GBC (OR 0.37) [54]. Several in vitro studies have been performed attempting to treat GBC with various anti-inflammatory agents with promising results [52], but there have been no human trials to date.

\section{Molecular pathogenesis and genomics of gallbladder cancer}

The pathological spectrum of progression in GBC involves various stages including metaplasia, dysplasia, carcinoma-in-situ and invasive cancer (Fig. 3) [6]. The most common pathway to GBC progression involves the intermediate stages of metaplasia and dysplasia. The classic adenoma-carcinoma sequence occurs in GBC with far less frequency $(<3 \%)$ [6]. The underlying somatic molecular changes of GBC remain ill-understood. However, based on previous studies we have a broad understanding of the commonly associated gene changes which are described below.

a. Somatic and copy number changes: A variety of genomic changes have been identified in GBC $[6,55]$. These include somatic mutations, microsatellite instability, loss of heterozygosity, gene overexpression, epigenetic changes and miRNA associated changes [55]. Different cell signaling pathways are implicated in the causation of biliary tract cancers and GBC. These include the $E r b B$ pathway [56, 57], PI3K/Akt/mTOR pathway [58], MAPK/ERK pathway [59], VEGF pathway [60], Notch pathway [61] and Hedgehog pathway [62] to name a few. GBC pathogenesis involves the complex interaction of various genomic changes at a DNA, RNA and epigenetic level. Li et al. performed the first comprehensive mutational profiling in GBC [63]. This study identified 1450 somatic changes at an exome level. The most commonly mutated genes included the TP53, KRAS, and the $E r b B$ pathway genes. An overrepresentation of somatic mutational alterations was seen in the $\operatorname{ErbB}$ signaling pathway, comprised of the EGFR, HER2, $E R B B 3$, and $E R B B 4$ genes, was seen in this study ( $n=$ 21/57) [63]. ErbB pathway genes are key growth factor receptor genes commonly implicated in multiple types of cancers. In addition, gene amplification of HER2 and overexpression of EGFR and HER2 has been observed in GBC in multiple other studies as well. In New Mexico, we observe HER2 overexpression in $25 \%$ of GBC cases $(n=6 / 25$, unpublished data, see Figs. 4 and 5).

Activation of KRAS is identified in a subset of GBCs, with increased activation seen in anomalous junction of the pancreaticobiliary duct (AJPBD). A study specifically 


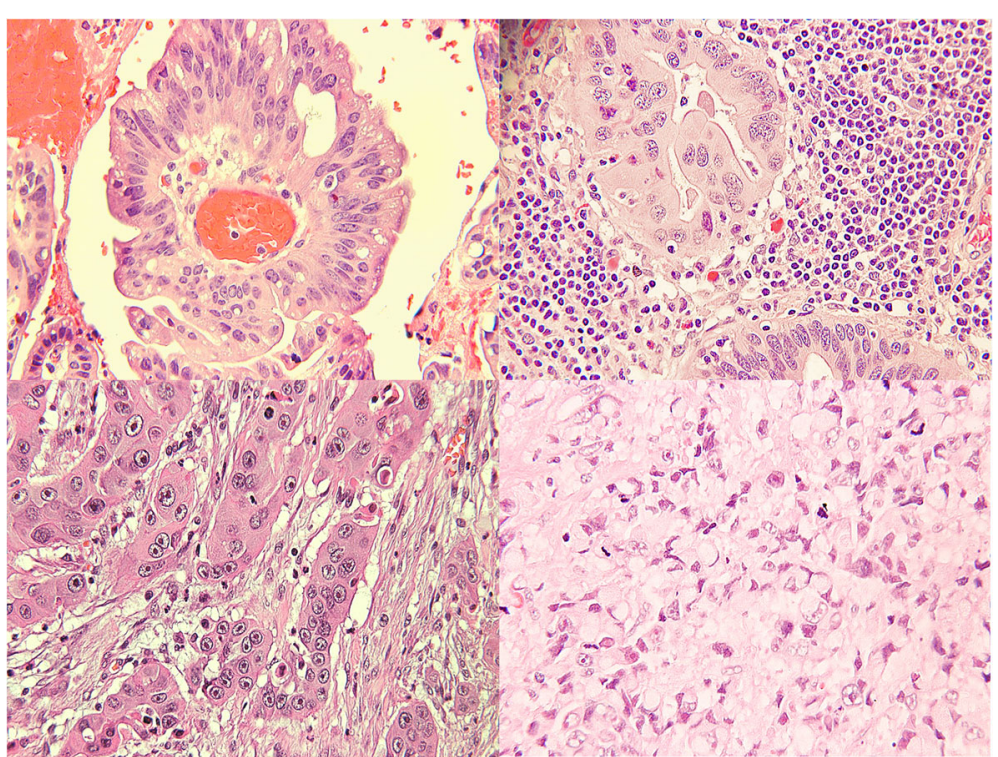

Fig. $3 \mathrm{~A}$ hematoxylin and eosin stained image of gallbladder cancer histomorphology. The four panels (clockwise from top left) shows the differing degrees of differentiation commonly observed in gallbladder cancer pathology specimens (dysplasia, well differentiated, poorly differentiated, and moderately differentiated)

looking at $B R A F$, which is a key effector of the oncologic activity of $K R A S$, found $B R A F$ mutations in $33 \%$ of GBCs studied [64]. Additionally, these mutations were found to be mutually exclusive, which has also been noted in other types of cancer. A recent systematic review and meta-analysis of genomic profiling identified 80 candidate gene variants and 173 polymorphisms associated with GBC in total [65]. Although this analysis found most of the studies to be of indeterminate quality due to the small sample sizes and lack of confirmatory testing, the following candidate genes were found to be significant: XPC, ERCC2, MSH2, OGG1, XRCC1, EGF, KRAS Gln25His, NAT2, GSTT1, ESR1, and CYP7A1 [65]. The meta-analysis found only the TP53 mutational changes to be significantly associated with GBC susceptibility [65].

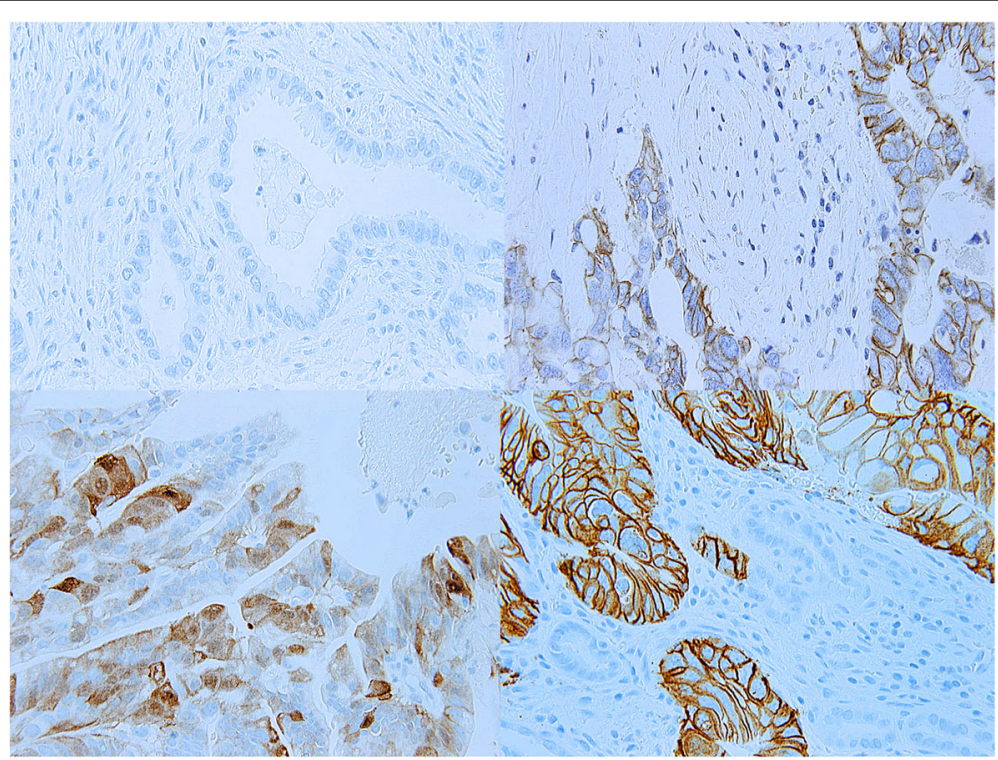

Fig. 4 HER2 positivity in New Mexican cases of gallbladder cancer by immunohistochemistry. The panels (clockwise from top left) represent HER2 staining status of $0,1+, 3+$ and $2+$ grades. Gallbladder cancer HER2 staining is axial, similar to the staining pattern seen in gastric cancers. In contrast, HER2 staining in breast cancers is more circumferential and evenly distributed around the tumor cell periphery 


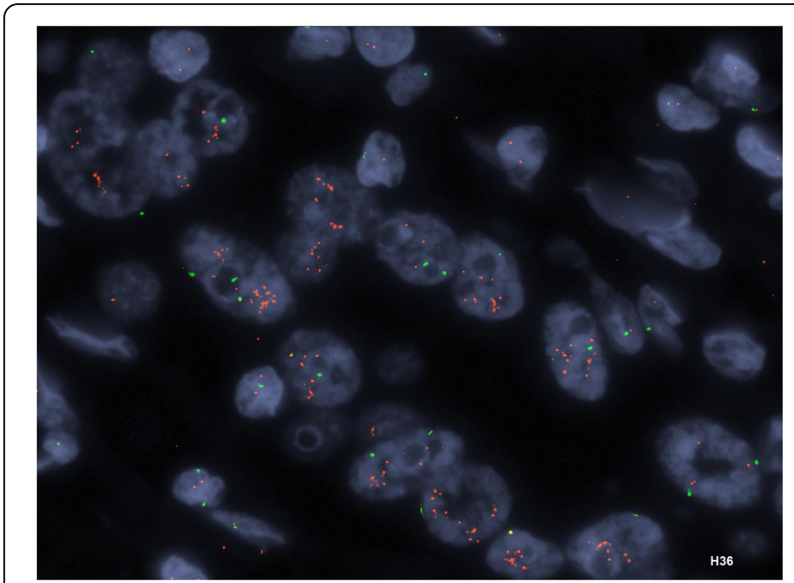

Fig. 5 HER2 gene amplification detected by Fluorescence In-Situ Hybridization (FISH) method. This case had approximately nine HER2 gene copies per cell and showed a concordant 3+ staining for HER2 protein expression by $\mathrm{IHC}$

b. Chromosome level changes: Allelic loss of heterozygosity has been observed in GBC at the $1 \mathrm{p}$, 3p, 8p, 9p, 13q, 16q, 17p and 22q locations of the chromosome [48]. Microsatellite instability (MSI) is observed in GBC at rates similar to other GI malignancies [66]. The incidence of MSI in GBC has been reported to be between 0 and $40 \%$ with an average of $\sim 10 \%$ of the GBC patients [66]. In our New Mexican GBC cohort, we observed an elevated tumor mutational burden (TMB) in $8 \%$ of our GBC cohort ( $n=2 / 25$, unpublished data) consistent with observations elsewhere. MSI and $\mathrm{TMB}$ are increasingly important as predictive biomarkers of response to immunotherapies. Epigenetic changes have also been linked to GBC pathogenesis. Altered methylation patterns of the $p 73, M G M T, M L H 1, A P C$ and $p 16$ genes has been identified in $72 \%$ of GBCs and $28 \%$ of chronic cholecystitis cases [67]. Epigenetic alternations of the DNA repair systems are implicated in the microsatellite instability seen in GBC patients.

In summary, like many other GI malignancies, the diversity of genomic alterations in GBC appears to be high and ill-defined with diverse mutational, copy number, chromosomal and epigenetic changes. The genomic changes in GBC are likely the result of a complex interactions due to the initiating chronic inflammation and environmentally driven factors over a prolonged period. The relative rarity of the tumor is an additional challenge to the detailed genomic level understanding of this form of cancer. Further studies (human and animal models) are urgently required to delineate the precise step-wise progression of genomic changes in GBC.

\section{Therapy considerations in native American and other rural, and medically underserved GBC populations.}

Two-thirds of GBC patients are diagnosed incidentally at the time of surgery for symptomatic cholelithiasis/cholecystitis [68]. Incidental diagnosis of GBC occurs in $0.2-3 \%$ of all cholecystectomies for presumed benign disease [69]. The remaining one third of patients present with symptoms from locally advanced disease, including right upper quadrant/epigastric pain, obstructive jaundice, nausea, anorexia and weight loss. Patients presenting with symptomatic disease tend to be locally advanced/metastatic at presentation and $75 \%$ are found with unresectable disease [70].

The standard of care for both curative and palliative treatment of GBC with regards to surgery, chemotherapy, and radiation are summarized in Table 1 . The treatment guidelines are broadly applicable to Native American patients and other minority, rural, and medically underserved patient groups. Yet, Native American communities have poorer cancer outcomes, suboptimal cancer screening, and high-risk cancer behaviors [71]. Native American cancer patients are less likely to undergo recommended cancer surgeries, adjuvant chemotherapy, and radiation therapy compared to the non-Native populations. Due to these factors, Native Americans have the worst cancer survival rates of any US ethnic group in general [72]. Despite these recognized health disparities, Native American patients with GBC did not demonstrate a significant differences in stage at diagnosis or survival compared to non-Native American populations [73]. This may be due to the advanced stage and poor prognosis associated with $\mathrm{GBC}$ in general, regardless of access to healthcare. There is a paucity of GBC treatment data in Native American populations. Access to specialized surgical care is critical in GBC management. Markin et al. evaluated whether operative outcomes contribute to poor long-term survival among Native American patients with cancer, including a small subset with GBC [74]. Their analysis demonstrated that Native American patients were younger, more likely to undergo cancer surgery at rural hospitals, and more likely to be admitted for non-elective procedures and had more comorbidities compared to non-Hispanic white patients of similar ages (all, $P<.05)$. Native American patients had comparable inpatient mortality and length of stay. The authors suggest that future research should focus on other cancer care delivery factors that may contribute to the poor long-term survival of Native American patients with cancer, including delivery of perioperative therapies.

The role of adjuvant therapy in GBC remains ill-defined, though it is recommended by both the National Comprehensive Cancer Network (NCCN) and the European Society of Medical Oncology (ESMO) for Stage I-III disease following surgery with curative intent (Table 1). Oral capecitabine, as administered in the BILCAP trial, is a 

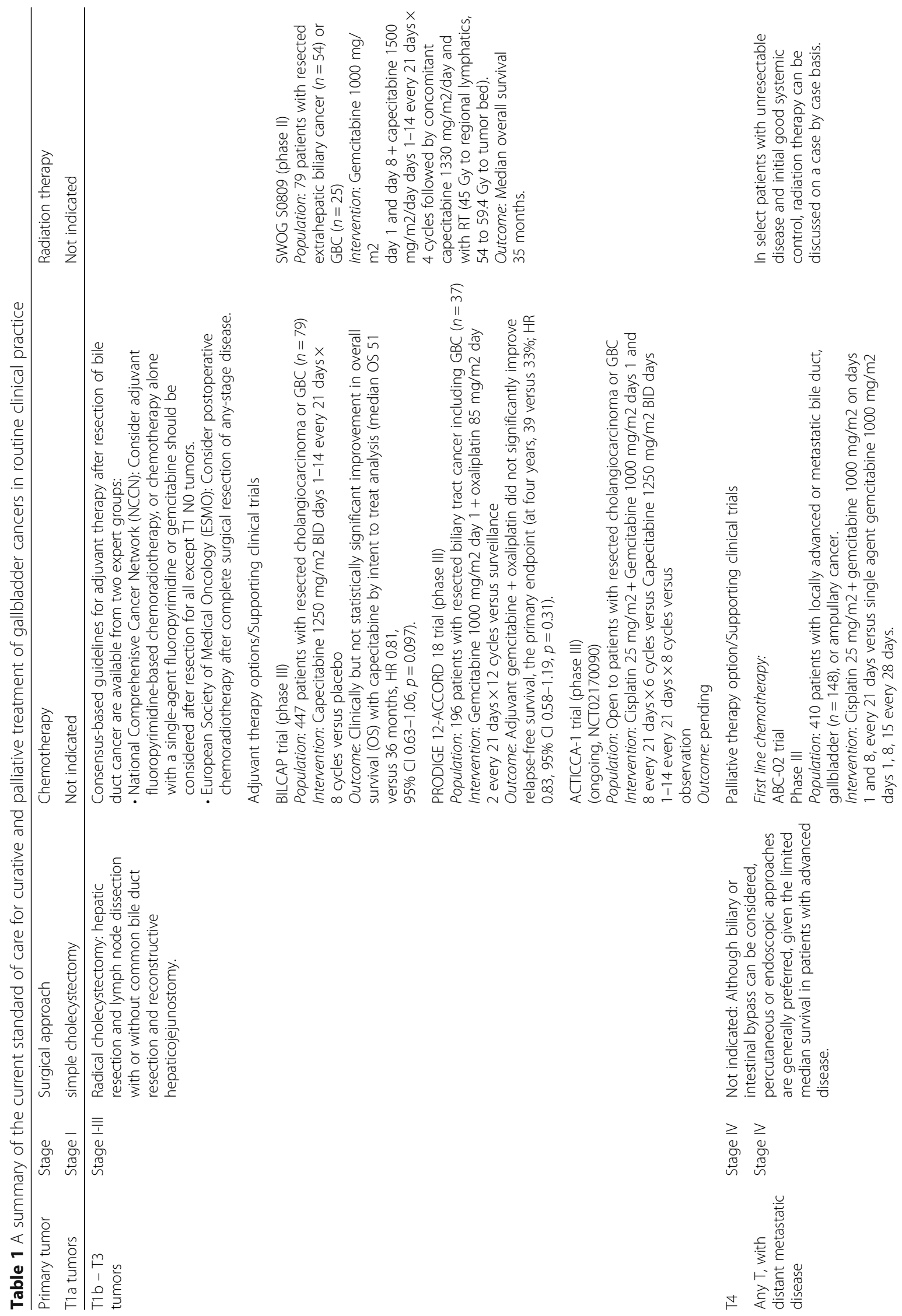

总意

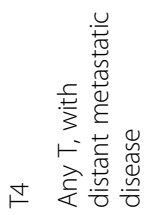




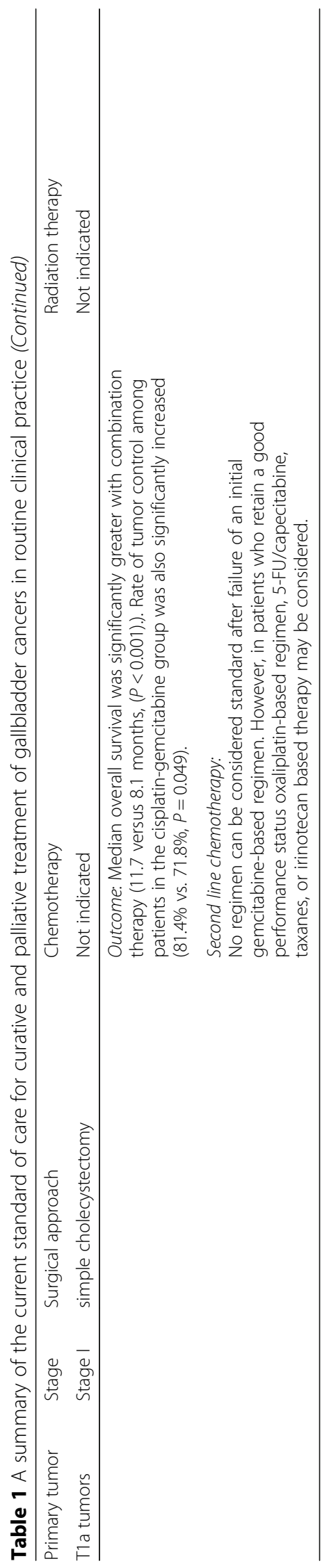


particularly appealing adjuvant therapy option for patients living in a rural setting far away from major treatment centers, as is the case for many New Mexican GBC patients. Adjuvant radiation therapy access remains a unique challenge for rural cancer patients. Athas et al. evaluated 1122 cases of localized breast cancer using the New Mexico NCI SEER Tumor Registry (NMTR) [75]. After adjusting for age, the likelihood of receiving radiation therapy following breast conserving surgery decreased significantly with increased travel distance to the nearest radiation oncology facility, with $51 \%$ of women living 75 miles or more from the closest facility receiving radiation compared with $82 \%$ residing within 50 miles travel distance. These findings highlight the difficulty rural cancer patients have accessing specialized cancer care including a New Mexico -centric cancer such as GBC.

Rural and underserved cancer patient populations also experience disparities in supportive palliative care services. This was demonstrated in a SEER analysis of Native American and White patients dying of breast, cervix, colorectal, kidney, lung, pancreas, prostate cancer, or stomach cancer from 2003 to 2009 [76]. In this analysis, a lower proportion of Native Americans enrolled in hospice compared to White patients ( $54 \%$ vs $65 \%$, respectively; $P<.0001)$. While the proportion of White patients who used hospice services in the last 6 months of life increased from $61 \%$ in 2003 to $68 \%$ in $2009(P<.0001)$, the proportion of American Indian patients using hospice care remained unchanged $(P=.57)$ and remained below that of their Caucasian counterparts throughout the study [76]. Telemedicine may serve as a potential solution to address these disparities. For example, Project ECHO (Extension for Community Healthcare Outcomes) originating at University of New Mexico, uses teleconferencing technology to support and train healthcare providers (HCPs) in remote rural locations. ECHO has been successfully used to provide education and support to community hospice nurses $[77,78]$. Our center is currently piloting the use of Project ECHO to facilitate complex cancer care and access to clinical trials to community oncology patients within the state of New Mexico. $\mathrm{GBC}$, a disease requiring access to multidisciplinary specialized care including medical and surgical oncology, radiation oncology, interventional radiology and gastroenterology, as well as palliative care, is a disease that will benefit from such an intervention.

\section{Prevention strategies in gallbladder cancers}

Given the fact that GBC is most frequently diagnosed when the disease is advanced and no longer curable, effective screening modalities are of critical importance. A successful screening strategy will require the identification of patients at significantly high risk for GBC based on the risk factors discussed above. Some practitioners recommend the use of prophylactic cholecystectomy in patients with silent gallstones in areas of high prevalence of disease. Empiric evidence suggests increased rates of cholecystectomies can have a significant effect on the rate of GBC. In India where there is a known high prevalence of GBC, it was found that 1 case of GBC could be prevented for every 67 laparoscopic cholecystectomies. Comparatively, in regions where the GBC is relatively low (e.g., continental US), one case of GBC is preventable for every 769 cholecystectomies [79]. In response to abnormally high incidence of GBC, the government of Chile instituted a program of prophylactic cholecystectomy in 2006 in people aged 35-49 years with gallstones which is currently underway. Yet, the health care costs associated with prospective surgical interventions (e.g., cholecystectomies) represent a significant financial burden in low-GDP countries like India and Chile, potentially limiting the scope of such approaches. Similarly, the risks associated with surgical interventions are significant. Lastly, given the inciting role for inflammation in this disease, NSAIDs may play a role in chemoprevention and requires further research.

\section{Future directions in the treatment of GBC}

Guideline based palliative systemic therapy for GBC is limited to cytotoxic chemotherapy (see Table 1). As with other malignancies, multiple efforts are underway globally to identify targeted agents for the treatment of GBC, though none are established as standard of care in routine clinical practice (see Table 2). Many of the clinical trials group biliary tract cancers together (intra-hepatic cholangiocarcinoma, extra-hepatic cholangiocarcinoma, and GBC), making stand-alone analysis of GBC difficult. We briefly describe some of the promising approaches below for GBC.

\section{HER2 and EGFR targeting therapies}

Targeting the HER2/EGFR family pathway has become increasingly attractive in a variety of gastrointestinal malignancies. The HER2 protein has been shown to be variably amplified in $16-64 \%$ of GBC [80, 81]. Similarly, EGFR amplifications and mutations have been described in 6 and 13.6\%-15\% respectively [82]. Unfortunately, early clinical trials to date have not demonstrated a significant benefit in targeting these pathways in isolation. In in-vitro studies with lapatinib, a dual tyrosine kinase inhibitor which interrupts both the HER2 and EGFR pathways, demonstrated a synergistic anti-tumor effect on GBC cell lines when combined with gemcitabine [83]. These promising pre-clinical results led to two phase II trials of single agent lapatinib in biliary tract cancers (including GBC). These trials were ultimately negative, however the study population was not selected for relevant HER2 amplification [82]. Another second case series followed 
Table 2 Current clinical trials in progress for targeted therapeutic options for gallbladder cancers (data from www.clinicaltrials.gov)

\begin{tabular}{|c|c|c|c|c|c|}
\hline Agent & Target & Regimen & Phase & Institution & $\mathrm{NCl}$ Number \\
\hline $\begin{array}{l}\text { Cetuximab, Trastuzumab, } \\
\text { Gefitinib, Lapatinib, Everolimus, } \\
\text { Sorafenib, Crizotinib }\end{array}$ & $\begin{array}{l}\text { EGFR, HER2, mTOR, } \\
\text { VEGF, ALKVROS1, } \\
\text { PDL-1 }\end{array}$ & $\begin{array}{l}\text { GEMOX + targeted therapy per } \\
\text { proteomic/genetic profiling }\end{array}$ & $\|$ & Xinhua Hospital, Shanghai & NCT02836847 \\
\hline Durvalumab & PDL-1 & Guadecitabine + Durvalumab & । & University of Southern California & NCT03257761 \\
\hline Pazopanib & CKIT, FGFR, VEGFR & Gemcitabine + Pazobanib & $\|$ & $\begin{array}{l}\text { Hellenic Cooperative Oncology } \\
\text { Group }\end{array}$ & NCT01855724 \\
\hline Pembrolizumab & PD-1 & $\begin{array}{l}\text { Pembrolizumab + Cisplatin + } \\
\text { Gemcitabine }\end{array}$ & $\|$ & $\begin{array}{l}\text { European Organization for Research } \\
\text { and Treatment of Cancer }\end{array}$ & NCT03260712 \\
\hline Selumetinib & MEK1, MEK2 & $\begin{array}{l}\text { Selumetinib + Cisplatin + } \\
\text { Gemcitabine }\end{array}$ & $\|$ & University Health Network, Toronto & NCT02151084 \\
\hline $\mathrm{ADH}-1$ & $\mathrm{~N}$-Cadherin & $\begin{array}{l}\text { ADH-1 + Cisplatin + } \\
\text { Gemcitabine }\end{array}$ & । & University of Nebraska & NCT01825603 \\
\hline Canlisib & PI3K & $\begin{array}{l}\text { Copanlisib + Cisplatin + } \\
\text { Gemcitabine }\end{array}$ & $\|$ & $\begin{array}{l}\text { H. Lee Moffitt Cancer Center } \\
\text { and Research Institute }\end{array}$ & NCT02631590 \\
\hline Merestinib & MET & $\begin{array}{l}\text { Merestinib + Cisplatin + } \\
\text { Gemcitabine }\end{array}$ & । & Eli Lilly and Company & NCT03027284 \\
\hline Regorafenib & VEGF & Regorafenib & $\|$ & $\begin{array}{l}\text { H. Lee Moffitt Cancer Center } \\
\text { and Research Institute }\end{array}$ & NCT02115542 \\
\hline
\end{tabular}

nine patients treated with HER2 directed therapy (trastuzumab, lapatinib, or pertuzumab) either alone or in combination with chemotherapy demonstrating one complete response, four partial responses, and three cases with stable disease [84]. One patient treated with lapatinib alone had mixed response, indicating a possible role of HER2 monotherapy. A phase II trial investigating trastuzumab in unresectable biliary tract cancer was closed prematurely due to difficulty with enrollment and lack of response signal (NCT00478140).

With regards to EGFR, a phase III trial evaluated the efficacy of erlotinib, a tyrosine kinase inhibitor targeting EFGR, combined with gemcitabine and oxaliplatin, in patients with advanced biliary tract cancer has been completed. Progression free survival was 5.8 months in the erlotinib group compared with 4.2 months, but overall survival was the same for both groups and no benefit was noted in GBC patients [85]. Several phase II trials investigating panitumumab, a monoclonal antibody targeted to EGFR, combined with chemotherapy in KRAS wild-type patients with biliary tract cancer have demonstrated mixed results, with the largest randomized trial showing no difference in survival [82]. Targeted inhibitors in combination with regular chemotherapy appears to show promise for future $\mathrm{GBC}$ treatments.

\section{Other therapeutic targets}

Several clinical trials have investigated the use of $V E G F$ inhibition in GBC. In a phase II trial in all biliary tract cancers, bevacizumab, a monoclonal antibody that inhibits $V E G F-A$, combined with gemcitabine and oxaliplatin, demonstrated a 6 month PFS of $63 \%$, which was below the target rate of $70 \%$ [86]. Another phase II study using cediranib, an oral VEGFR1, VEGFR2, and VEGF3 tyrosine kinase inhibitor, similarly failed to meet its primary endpoint of improvement in median PFS [87]. Studies investigating antiangiogenic tyrosine kinase inhibitors sorafenib and sunitinib have been similarly disappointing [82]. Other pathways of interest for targeting purposes in GBC include $A P C, B R A F, K R A S$, and P53. Similarly, immunotherapy has shown promising signals in GBC. In a study of biliary tract cancer patients, $42 \%$ of 89 patients were found to be PD-L1 positive [82]. Numerous ongoing clinical trials utilizing immune checkpoint inhibitors include subsets of biliary tract cancer. Of particular interest is a study at Xinhua Hospital in Shanghai that seeks to assign targeted therapy to biliary tract cancer patients according to relevant mutations (NCT02836847). Results from this and several other trials are pending. Table 2 provides a summary of ongoing clinical trials with targeted therapies for GBC. There is much excitement for the potential of targeted drug and immunotherapy based approaches in GBC treatment, through much pre-clinical and clinical work remains to be done to demonstrate the enhanced efficacy of these newer therapeutic approaches.

\section{Conclusions}

GBC is a relatively rare disease with a poor clinical prognosis and with a unique geographic distribution and risk factor profile. In the United States, Native American populations in the Southwest are uniquely impacted. The elevated incidence of gallstones among Native Americans is likely to be the major driving factor of increased of $\mathrm{GBC}$ in this population. Unfortunately, 
treatment options for this disease are still quite limited. Surgical resections, cytotoxic chemotherapy and radiation are the mainstay of GBC treatments, all with modest survival benefit. Research efforts are currently underway to understand the molecular pathogenesis of GBC, which may aid in identifying targeted therapeutic approaches. The difficulty in treating GBC is in part due to the relative rarity of the tumor and the lack of robust basic research efforts. The lack of readily available animal models of GBC also presents a significant hurdle to our understanding. Lastly, many GBC patients present at an advanced stage with a poor performance status, rendering them ineligible for clinical trial participation. Given the high mortality and lack of therapeutic options, GBC patients will benefit from enhanced efforts at developing pre-clinical research with subsequent translation to therapeutic clinical trials with the goal of improving survival outcomes.

\section{Abbreviation \\ GBC: Gallbladder Cancer}

\section{Funding}

RRG was supported by the Institutional Development Award (IDeA) from the National Institute of General Medical Sciences of the National Institutes of Health under grant number P20GM103451 and start-up funds from the University of New Mexico, Department of Pathology.

\section{Authors' contributions}

$J M N$, UBG and RRG wrote the original draft of the paper. HS, JB, BL, IN, VP reviewed the paper and suggested draft changes. All authors read and approved the final version of the manuscript.

\section{Ethics approval and consent to participate}

Not applicable.

\section{Consent for publication}

Not applicable.

\section{Competing interests}

RRG currently serves as an associate editor of the BMC Cancer Journal.

\section{Publisher's Note}

Springer Nature remains neutral with regard to jurisdictional claims in published maps and institutional affiliations.

\section{Author details \\ 'Comprehensive Cancer Center, University of New Mexico, Albuquerque, NM, USA. ${ }^{2}$ Department of Internal Medicine, Division of Hematology and Oncology, University of New Mexico Health Sciences Center, Albuquerque, NM, USA. ${ }^{3}$ Department of Surgery, Division of Surgical Oncology, University of New Mexico Health Sciences Center, Albuquerque, NM, USA. " Department of Pathology, University of New Mexico Health Sciences Center, Albuquerque, NM, USA. ${ }^{5}$ Department of Chemical and Biological Engineering, University of New Mexico, Room 333A, MSC08-4640, Albuquerque, NM 87131, USA.}

\section{Received: 9 March 2018 Accepted: 1 June 2018}

Published online: 18 June 2018

\section{References}

1. Wistuba II, Gazdar AF. Gallbladder cancer: lessons from a rare tumour. Nat Rev Cancer. 2004;4(9):695-706

2. Hundal R, Shaffer EA. Gallbladder cancer: epidemiology and outcome. Clin Epidemiol. 2014;6:99-109.
3. Shaffer EA. Gallbladder cancer: the basics. Gastroenterol Hepatol (N Y). 2008; 4(10):737-41.

4. Nir I, Wiggins CL, Morris K, Rajput A. Diversification and trends in biliary tree cancer among the three major ethnic groups in the state of New Mexico. Am J Surg. 2012;203(3):361-5. discussion 365

5. Roa I, de Aretxabala X. Gallbladder cancer in Chile: what have we learned? Curr Opin Gastroenterol. 2015;31(3):269-75.

6. Bizama C, Garcia P, Espinoza JA, Weber H, Leal P, Nervi B, et al. Targeting specific molecular pathways holds promise for advanced gallbladder cancer therapy. Cancer Treat Rev. 2015;41(3):222-34.

7. Randi G, Franceschi S, La Vecchia C. Gallbladder cancer worldwide: geographical distribution and risk factors. Int J Cancer. 2006;118(7): 1591-602.

8. Lemrow SM, Perdue DG, Stewart SL, Richardson LC, Jim MA, French HT, et al. Gallbladder cancer incidence among American Indians and Alaska natives, US, 1999-2004. Cancer. 2008;113(5 Suppl):1266-73.

9. Lorenzo Bermejo J, Boekstagers F, Gonzalez Silos R, Marcelain K, Baez Benavidez P, Barahona Ponce C, et al. Subtypes of native American ancestry and leading causes of death: Mapuche ancestry-specific associations with gallbladder cancer risk in Chile. PLoS Genet. 2017;13(5):e1006756.

10. Diehl AK, Beral V. Cholecystectomy and changing mortality from gallbladder cancer. Lancet. 1981;2(8239):187-9.

11. Koshiol J, Wozniak K, Cook P, Adaniel C, Acevedo J, Azocar L, et al. Salmonella enterica serovar Typhi and gallbladder cancer: a case-control study and meta-analysis. Cancer Med. 2016;5(11):3310-235.

12. Nagaraja V, Eslick GD. Systematic review with meta-analysis: the relationship between chronic Salmonella typhi carrier status and gall-bladder cancer. Aliment Pharmacol Ther. 2014;39(8):745-50.

13. Fox JG, Dewhirst FE, Shen Z, Feng Y, Taylor NS, Paster BJ, et al. Hepatic helicobacter species identified in bile and gallbladder tissue from Chileans with chronic cholecystitis. Gastroenterology. 1998;114(4):755-63.

14. Mendez-Sanchez N, Pichardo R, Gonzalez J, Sanchez H, Moreno M, Baquero $F$, et al. Lack of association between helicobacter sp colonization and gallstone disease. J Clin Gastroenterol. 2001;32(2):138-41.

15. Khan ZS, Livingston EH, Huerta S. Reassessing the need for prophylactic surgery in patients with porcelain gallbladder: case series and systematic review of the literature. Arch Surg. 2011;146(10):1143-7.

16. Redaelli CA, Buchller MW, Schilling MK, Krahenbuhl L, Ruchti C, Blumgart H, et al. High coincidence of Mirizzi syndrome and gallbladder carcinoma. Surgery. 1997;121(1):58-63.

17. Pilgrim CH, Satgunaseelan L, Ward SM, Evans PM. Gallbladder carcinoma as a long-term complication of cholecystojejunostomy. J Gastrointest Surg. 2009;13(12):2330-2.

18. Foerster C, Koshiol J, Guerrero A, Kogan M, Ferrecio C. The case for aflatoxins in the causal chain of gallbladder cancer. Med Hypotheses. 2016;86:47-52.

19. Koshiol J, Gao YT, Dean M, Eager P, Nepal C, Jones K, et al. Association of Aflatoxin and Gallbladder Cancer. Gastroenterology. 2017;153(2):488-494 e1.

20. Cirillo DJ, Wallace RB, Rodabough RJ, Greenland P, LaCroix AZ, Limacher MC, et al. Effect of estrogen therapy on gallbladder disease. JAMA. 2005;293(3): 330-9.

21. Eslick GD. Epidemiology of gallbladder cancer. Gastroenterol Clin N Am. 2010;39(2):307-30. ix

22. Shukla VK, Chauhan VS, Mishra RN, Basu S. Lifestyle, reproductive factors and risk of gallbladder cancer. Singap Med J. 2008;49(11):912-5.

23. Liu H, Ziang Y, Ai M, Wang J, Jin B, Wo M, et al. Body mass index can increase the risk of gallbladder Cancer: a meta-analysis of 14 cohort studies. Med Sci Monit Basic Res. 2016;22:146-55.

24. Gälman C, Miquel JF, Perez RM, Einaressen C, Stahle L, Marshall G, et al. Bile acid synthesis is increased in chilean hispanics with gallstones and in gallstone high-risk Mapuche Indians. Gastroenterology. 2004;126(3):741-8.

25. Carey MC, Paigen B. Epidemiology of the American Indians' burden and its likely genetic origins. Hepatology. 2002;36(4 Pt 1):781-91.

26. Sievers ML, Marquis JR. The southwestern American Indian's burden: biliary disease. JAMA. 1962;182:570-2.

27. Grundy SM, Metzger AL, Adler RD. Mechanisms of lithogenic bile formation in American Indian women with cholesterol gallstones. J Clin Invest. 1972; 51(12):3026-43.

28. Jaruvongvanich $V$, Yang JD, Peeraphatdit T, Roberts LR. The incidence rates and survival of gallbladder cancer in the USA. Eur J Cancer Prev. 2017. PMID: 28683010. https://doi.org/10.1097/CEJ.0000000000000402. 
29. Pandey M. Environmental pollutants in gallbladder carcinogenesis. J Surg Oncol. 2006;93(8):640-3.

30. Nath G, Gulati AK, Shukla VK. Role of bacteria in carcinogenesis, with special reference to carcinoma of the gallbladder. World J Gastroenterol. 2010; 16(43):5395-404

31. Chhabra D, Oda K, Jagannath $P$, Utsunomiya H, Takekoshi S, Nimura Y. Chronic heavy metal exposure and gallbladder Cancer risk in India, a comparative study with Japan. Asian Pac J Cancer Prev. 2012;13(1):187-90.

32. Blake JM, Avasarala S, Artyushkova K, Ali A, Brearley A, Shuey C, et al. Elevated concentrations of $U$ and co-occurring metals in abandoned mine wastes in a northeastern Arizona native American community. Environ Sci Technol. 2015;49(14):8506-14.

33. Harmon ME, Lewis J, Miller C, Hoover J, Ali AS, Shuey C, et al. Residentia proximity to abandoned uranium mines and serum inflammatory potentia in chronically exposed Navajo communities. J Expo Sci Environ Epidemiol. 2017;27(4):365-71.

34. Hoover J, Gonzales M, Shuey C, Barney Y, Lewis J. Elevated arsenic and uranium concentrations in unregulated water sources on the Navajo nation, USA. Expo Health. 2017:9(2):113-24.

35. Briner W. The toxicity of depleted uranium. Int J Environ Res Public Health. 2010;7(1):303-13.

36. Hao Y, Huang J, Gu Y, Liu C, Li H, Liu J, et al. Metallothionein deficiency aggravates depleted uranium-induced nephrotoxicity. Toxicol Appl Pharmacol. 2015:287(3):306-15.

37. Espinoza JA, Bizama C, Garcia P, Ferreccio P, Javle M, Miguel JF, et al. The inflammatory inception of gallbladder cancer. Biochim Biophys Acta. 2016;1865(2):245-54.

38. Li Y, Zhang J, Ma H. Chronic inflammation and gallbladder cancer. Cancer Lett. 2014;345(2):242-8.

39. Aleksandrova $K$, Boeing $H$, Nothlings $U$, Jenab $M$, Fedirko $V$, Kaaks $R$, et al. Inflammatory and metabolic biomarkers and risk of liver and biliary tract cancer. Hepatology. 2014;60(3):858-71.

40. Hussain SP, Harris CC. Inflammation and cancer: an ancient link with novel potentials. Int J Cancer. 2007;121(11):2373-80.

41. Shi JS, Zhou LS, Han Y, Zhu AJ, Sun XJ, Yang YJ. Expression of tumor necrosis factor and its receptor in gallstone and gallbladder carcinoma tissue. Hepatobiliary Pancreat Dis Int. 2004;3(3):448-52.

42. Lee HJ, Lee K, Lee DJ, Bae KH, Kim JS, Liang ZL, et al. Chemokine (C-X-C motif) ligand 12 is associated with gallbladder carcinoma progression and is a novel independent poor prognostic factor. Clin Cancer Res. 2012;18(12):3270-80.

43. Yao X, Zhou L, Han S, Chen Y. High expression of CXCR4 and CXCR7 predicts poor survival in gallbladder cancer. J Int Med Res. 2011;39(4):1253-64.

44. Hayashi, N., Yamamoto, H., Hiraoka, N., Dono, K., Ito, Y., Okami, J., et al., Differential expression of cyclooxygenase-2 (COX-2) in human bile duct epithelial cells and bile duct neoplasm. Hepatology, 2001. 34(4 Pt 1): p. 638-650.

45. Srivastava K, Srivastava A, Kumar A, Mittal B. Significant association between toll-like receptor gene polymorphisms and gallbladder cancer. Liver Int. 2010;30(7):1067-72.

46. Srivastava A, Pandey SN, Choudhuri G, Mittal B. CCR5 Delta32 polymorphism: associated with gallbladder cancer susceptibility. Scand Immunol. 2008;67(5):516-22.

47. Moreno M, Pimental F, Gasdar AF, Wistuba II, Miguel JF. TP53 abnormalities are frequent and early events in the sequential pathogenesis of gallbladder carcinoma. Ann Hepatol. 2005;4(3):192-9.

48. Jain K, Mahapatra T, Das P, Misra MC, Gupta SD, Ghosh M, et al. Sequential occurrence of preneoplastic lesions and accumulation of loss of heterozygosity in patients with gallbladder stones suggest causal association with gallbladder cancer. Ann Surg. 2014;260(6):1073-80.

49. Hsing AW, Sakoda LC, Rashid A, Andreotti G, Chen J, Weng BS, et al. Variants in inflammation genes and the risk of biliary tract cancers and stones: a population-based study in China. Cancer Res. 2008;68(15):6442-52.

50. Okayama H, Schetter AJ, Harris CC. MicroRNAs and inflammation in the pathogenesis and progression of colon cancer. Dig Dis. 2012;30(Suppl 2):9-15.

51. Merhautova J, Demlova R, Slaby O. MicroRNA-based therapy in animal models of selected gastrointestinal cancers. Front Pharmacol. 2016;7:329.

52. Deng M, Qin Y, Chen X, Li D, Wang Q, Zheng H, et al. Combination of celecoxib and PD184161 exerts synergistic inhibitory effects on gallbladder cancer cell proliferation. Oncol Lett. 2017;13(5):3850-8.

53. Kiguchi K, et al. Therapeutic effect of CS-706, a specific cyclooxygenase-2 inhibitor, on gallbladder carcinoma in BK5.ErbB-2 mice. Mol Cancer Ther. 2007;6(6):1709-17.
54. Liu E, Ruffino L, Kawamoto T, Franco E, Kurakata S, Fujiwara K, et al. Aspirin use and risk of biliary tract cancer: a population-based study in shanghai, China. Cancer Epidemiol Biomark Prev. 2005;14(5):1315-8.

55. Sharma A, Sharma KL, Gupta A, Yadav A, Kumar A. Gallbladder cancer epidemiology, pathogenesis and molecular genetics: recent update. World J Gastroenterol. 2017;23(22):3978-98.

56. Nakazawa K, Dobashi Y, Suzuki S, Fuji H, Takeda Y, Ooi A. Amplification and overexpression of c-erbB-2, epidermal growth factor receptor, and c-met in biliary tract cancers. J Pathol. 2005;206(3):356-65.

57. Ooi A, Suzuki S, Nakazawa K, Ikatura J, Imoto I, Nakamura H, et al. Gene amplification of Myc and its coamplification with ERBB2 and EGFR in gallbladder adenocarcinoma. Anticancer Res. 2009;29(1):19-26.

58. Leal, P., Garcia, P., Sandoval, A., Letelier,P., Brebi, P., Ili, C., et al., Immunohistochemical expression of phospho-mTOR is associated with poor prognosis in patients with gallbladder adenocarcinoma. Arch Pathol Lab Med, 2013. 137(4): p. 552-557.

59. Li Q, Yang Z. Expression of phospho-ERK1/2 and PI3-K in benign and malignant gallbladder lesions and its clinical and pathological correlations. J Exp Clin Cancer Res. 2009;28:65.

60. Letelier P, Garcia P, Leal P, Ili C, Buchegger K, Riquelme I, et al. Immunohistochemical expression of vascular endothelial growth factor a in advanced gallbladder carcinoma. Appl Immunohistochem Mol Morphol. 2014;22(7):530-6.

61. Yoon HA, et al. Clinicopathological significance of altered notch signaling in extrahepatic cholangiocarcinoma and gallbladder carcinoma. World J Gastroenterol. 2011:17(35):4023-30.

62. Xie F, Non MH, Kim BG, Han JS, Jang JS, Choi SR, et al. Aberrant activation of sonic hedgehog signaling in chronic cholecystitis and gallbladder carcinoma. Hum Pathol. 2014;45(3):513-21.

63. Li M, Zhang Z, Li X, Tan Z, Liu C, Shen B, et al. Whole-exome and targeted gene sequencing of gallbladder carcinoma identifies recurrent mutations in the ErbB pathway. Nat Genet. 2014;46(8):872-6.

64. Saetta AA, et al. Mutational analysis of BRAF in gallbladder carcinomas in association with K-ras and p53 mutations and microsatellite instability. Virchows Arch. 2004:445(2):179-82

65. Srivastava K, Papanastasiou P, Michalopoulos NV, Gigelou F, Korkoloupolou $P, B e i \mathrm{~T}$, et al. Candidate gene studies in gallbladder cancer: a systematic review and meta-analysis. Mutat Res. 2011;728(1-2):67-79.

66. Roa JC, Roa I, Correa P, Vo Q, Araya JC, Villaseca M, et al. Microsatellite instability in preneoplastic and neoplastic lesions of the gallbladder. J Gastroenterol. 2005;40(1):79-86.

67. Roa JC, Anabolon L, Roa I, Melo A, Araya JC, Tapia O, et al. Promoter methylation profile in gallbladder cancer. J Gastroenterol. 2006;41(3):269-75.

68. Jin K, Lan H, Zhu T, He K, Teng L. Gallbladder carcinoma incidentally encountered during laparoscopic cholecystectomy: how to deal with it. Clin Transl Oncol. 2011;13(1):25-33.

69. Fuks D, Regimbeau JM, Pessaux P, Bachellier P, Raventos A, Mantion G, et al. Is port-site resection necessary in the surgical management of gallbladder cancer? J Visc Surg. 2013;150(4):277-84.

70. Miller G, Jarnagin WR. Gallbladder carcinoma. Eur J Surg Oncol. 2008;34(3):306-12.

71. Guadagnolo BA, Petereit DG, Coleman CN. Cancer care access and outcomes for American Indian populations in the United States: challenges and models for progress. Semin Radiat Oncol. 2017;27(2):143-9.

72. Clegg LX, Li FP, Hankey BF, Chu K, Edwards BK, et al. Cancer survival among US whites and minorities: a SEER (surveillance, epidemiology, and end results) program population-based study. Arch Intern Med. 2002;162(17):1985-93.

73. Black WC, Key CR, Carmany TB, Herman D. Carcinoma of the gallbladder in a population of southwestern American Indians. Cancer. 1977;39(3):1267-79.

74. Markin A, Habberman EB, Zhu Y, Abraham A, Ahluwalia JS, Vickers SM, et al. Cancer surgery among American Indians. JAMA Surg. 2013;148(3):277-84. discussion 284

75. Athas WF, Cameron M, Hunt W, Amir-Fazli A, Key C. Travel distance to radiation therapy and receipt of radiotherapy following breast-conserving surgery. J Natl Cancer Inst. 2000:92(3):269-71.

76. Guadagnolo BA, Huo J, Buchholz TA, Petereit DG. Disparities in hospice utilization among American Indian Medicare beneficiaries dying of cancer. Ethn Dis. 2014;24(4):393-8.

77. White C, Mcllfatrick S, Dunwoody L, Watson M. Supporting and improving community health services-a prospective evaluation of ECHO technology in community palliative care nursing teams. BMJ Support Palliat Care. 2015.

78. Arora, S., Smith, T., Snead, J., Zalud-Cerrato, S., Marr, L., Watson, M., et al., Project ECHO: an effective means of increasing palliative care capacity. Am J Manag Care, 2017. 23(7 Spec No): p. SP267-SP271. 
79. Mathur AV. Need for prophylactic cholecystectomy in silent gall stones in North India. Indian J Surg Oncol. 2015;6(3):251-5.

80. Maurya SK, Tewari M, Mishra R, Shukla HS. Genetic aberrations in gallbladder cancer. Surg Oncol. 2012;21(1):37-43.

81. Muller BG, De Aretxabala X, Gonzalez Domingo M. A review of recent data in the treatment of gallbladder cancer: what we know, what we do, and what should be done. Am Soc Clin Oncol Educ Book. 2014:e165-70.

82. Valle JW, Lamarca A, Goyal L, Barriuso J, Zhu AX. New horizons for precision medicine in biliary tract cancers. Cancer Discov. 2017;7(9):943-62.

83. Pignochino Y, Sarotto I, Peraldo-Neia C, Penachioni JY, Cavalloni G, Migliardi $\mathrm{G}$, et al. Targeting EGFR/HER2 pathways enhances the antiproliferative effect of gemcitabine in biliary tract and gallbladder carcinomas. BMC Cancer. 2010;10:631.

84. Javle M, Churi C, Kang HC, Schroff R, Janku F, Surapaneni R, et al. HER2/neudirected therapy for biliary tract cancer. J Hematol Oncol. 2015;8:58.

85. Lee J, Park SH, Chang HM, Kim JS, Choi HJ, Lee MA, et al. Gemcitabine and oxaliplatin with or without erlotinib in advanced biliary-tract cancer: a multicentre, open-label, randomised, phase 3 study. Lancet Oncol. 2012; 13(2):181-8.

86. Zhu AX, Meyerhardt JA, Blaszkowsky LS, Kamdadakone AR, Muzikansky AR, Zheng $\mathrm{H}$, et al. Efficacy and safety of gemcitabine, oxaliplatin, and bevacizumab in advanced biliary-tract cancers and correlation of changes in 18-fluorodeoxyglucose PET with clinical outcome: a phase 2 study. Lancet Oncol. 2010;11(1):48-54.

87. Valle JW, Wasan H, Lopes A, Backen AC, Palmer DH, Morris K, et al. Cediranib or placebo in combination with cisplatin and gemcitabine chemotherapy for patients with advanced biliary tract cancer (ABC-03): a randomised phase 2 trial. Lancet Oncol. 2015;16(8):967-78.

\section{Ready to submit your research? Choose BMC and benefit from:}

- fast, convenient online submission

- thorough peer review by experienced researchers in your field

- rapid publication on acceptance

- support for research data, including large and complex data types

- gold Open Access which fosters wider collaboration and increased citations

- maximum visibility for your research: over $100 \mathrm{M}$ website views per year 